\title{
A global analysis of terrestrial plant litter dynamics in non- perennial waterways
}

Datry T. ${ }^{1,2 *}$, Foulquier A. ${ }^{3}$, Corti R. ${ }^{1}$, von Schiller D. ${ }^{4}$, Tockner K. ${ }^{5,6}$, Mendoza-Lera C. ${ }^{1}$, Clément J.C. ${ }^{7}$, Gessner M.O. ${ }^{5,8}$, Moleón M. ${ }^{9}$, Stubbington R. ${ }^{10}$, Gücker B. ${ }^{11}$, Albariño R. ${ }^{12}$, Allen D.C. ${ }^{13}$, Altermatt F. ${ }^{14}$, Arce M.I. ${ }^{4}$, Arnon S. ${ }^{15}$, Banas D. ${ }^{16}$, Banegas-Medina A. ${ }^{17}$, Beller E. ${ }^{18}$, Blanchette M.L. ${ }^{19}$, Blanco-Libreros J.F. ${ }^{20}$, Blessing J.J. ${ }^{21}$, Boëchat I.G. ${ }^{22}$, Boersma K.S. ${ }^{23}$, Bogan M.T. ${ }^{24}$, Bonada N..$^{25}$, Bond N.R. ${ }^{26}$, Brintrup Barría K.C. ${ }^{27}$, Bruder A. ${ }^{28}$, Burrows R.M. ${ }^{29}$, Cancellario T. ${ }^{30}$, Canhoto C..$^{31}$, Carlson S.M. ${ }^{32}$, Cauvy-Fraunié S. ${ }^{1}$, Cid N. ${ }^{25}$, Danger M. ${ }^{33}$, de Freitas Terra B. ${ }^{34}$, De Girolamo A.M. ${ }^{35}$, de La Barra E. ${ }^{36}$, del Campo R. ${ }^{37}$, Diaz-Villanueva V.D. ${ }^{2}$, Dyer F. ${ }^{38}$, Elosegi A. ${ }^{4}$, Faye E. ${ }^{39}$, Febria C. ${ }^{40}$, Four B. ${ }^{41}$, Gafny S. ${ }^{42}$, Ghate S.D. ${ }^{43}$, Gómez R. $^{37}$, Gómez-Gener L. ${ }^{44}$, Graça M.A.S. ${ }^{45}$, Guareschi S. ${ }^{37}$, Hoppeler F. ${ }^{46}$, Hwan J. ${ }^{24}$, Jones J.I. ${ }^{47}$, Kubheka S. ${ }^{48}$, Laini A. ${ }^{49}$, Langhans S.D. ${ }^{5}$, Leigh C. ${ }^{29}$, Little C.J..$^{50}$, Lorenz S..$^{51}$, Marshall J.C..$^{21}$, Martín E. ${ }^{50}$, McIntosh A.R..$^{40}$, Meyer E.I. ${ }^{52}$, Miliša M. ${ }^{53}$, Mlambo M.C..$^{54}$ Morais M. ${ }^{55}$, Moya N. ${ }^{56}$, Negus P.M. ${ }^{21}$, Niyogi D.K. ${ }^{57}$, Papatheodoulou A. ${ }^{58}$, Pardo I. ${ }^{59}$, Pařil P. ${ }^{60}$, Pauls S.U. ${ }^{46}$, Pešić V. ${ }^{61}$, Polášek M. ${ }^{60}$, Robinson C.T. ${ }^{50}$, Rodríguez-Lozano P. ${ }^{32}$, Rolls R.J. ${ }^{38}$, Sánchez-Montoya M.M. ${ }^{37}$, Savić A. ${ }^{62}$, Shumilova O. ${ }^{5}$, Sridhar K.R. ${ }^{43}$, Steward A.L. ${ }^{21}$, Storey R. ${ }^{63}$, Taleb A. ${ }^{64}$, Uzan A. ${ }^{65}$, Vander Vorste R. ${ }^{66}$, Waltham N.J. ${ }^{67}$, Woelfle-Erskine C. ${ }^{24}$, Zak D. ${ }^{67}$, Zarfl C. ${ }^{68}$ and Zoppini A. ${ }^{35}$

${ }^{1}$ UR RiverLy, centre de Lyon-Villeurbanne, 5 rue de la Doua CS 20244, 69625 Villeurbanne, France ${ }^{2}$ UMR "BOREA" CNRS 7208/IRD 207/MNHN/UPMC, DMPA, Museum National d'Histoire Naturelle, Paris Cedex, France. ${ }^{3}$ Université Grenoble Alpes, Laboratoire d'Écologie Alpine (LECA), UMR CNRS-UGA-USMB 5553, Grenoble, France. ${ }^{4}$ Department of Plant Biology and Ecology, Faculty of Science and Technology, University of the Basque Country (UPV/EHU), P.O. Box 644, 48080-Bilbao, Spain. ${ }^{5}$ Leibniz-Institute of Freshwater Ecology and Inland Fisheries (IGB), Berlin, Germany. ${ }^{6}$ Institute of Biology, Freie Universität Berlin, Germany.

${ }^{7}$ Université Savoie Mont Blanc, INRA, CARRTEL, 74200, Thonon-Les Bains, France. ${ }^{8}$ Department of Ecology, Berlin Institute of Technology (TU Berlin), Ernst-Reuter-Platz 1, 10587 Berlin, Germany. ${ }^{9}$ Department of Zoology, University of Granada, Avda. de Fuente Nueva, s/n, 18071-Granada, Spain. ${ }^{10}$ School of Science and Technology, Nottingham Trent University, UK. ${ }^{11}$ Department of Geosciences, Federal University of São João del-Rei, Campus Tancredo Neves, 36301-360 São João del-Rei, MG, Brazil. ${ }^{12}$ Laboratorio de Fotobiología, INIBIOMA (U.N.COMAHUE - CONICET), Bariloche, Argentina. ${ }^{13}$ University of Oklahoma, Department of Biology, Norman, OK, 73019 USA. ${ }^{14}$ Department of Evolutionary Biology and Environmental Studies, University of Zurich, Winterthurerstr. 190, CH-8057 Zürich, Switzerland. ${ }^{15}$ Zuckerberg Institute for Water Research, The Jacob Blaustein Institutes for Desert Research, Ben-Gurion University of the Negev, Sede Boqer, 84990, Israel. ${ }^{16}$ Université de Lorraine - UR AFPA, 54505 Vandoeuvre-Les-Nancy, France. ${ }^{17}$ Department of Aquatic Systems, Faculty of Environmental Science and EULA Chile Centre, Universidad de Concepción, Casilla 160-C, Concepción, Chile. ${ }^{18}$ Department of Geography, University of California, Berkeley, CA 94720, USA. ${ }^{19}$ Edith Cowan University, School of Science, Mine Water and Environment Research Centre (MiWER), Australia. ${ }^{20}$ Instituto de Biología, Universidad de Antioquia, Medellín, Colombia. ${ }^{21}$ Department of Science, Information Technology and Innovation, Queensland Government, Australia. ${ }^{22}$ Department of Geosciences, Federal University of São João del-Rei, Campus Tancredo Neves, 36301-360 São João del-Rei, MG, Brazil. ${ }^{23}$ University of San Diego, Department of Biology, San Diego, CA 92110, USA. ${ }^{24}$ School of Natural Resources and the Environment, University of Arizona, 1064 E lowelll street room N326 Tucson, AZ 85721, USA. ${ }^{25}$ Grup de Recerca Freshwater Ecology and Management (FEM), Departament de Biologia Evolutiva, Ecologia i Ciències Ambientals, Institut de Recerca de la Biodiversitat (IRBio), Universitat de Barcelona (UB), Diagonal 643, 08028-Barcelona, Catalonia, Spain. ${ }^{26}$ Murray-Darling Freshwater Research Centre, La Trobe University, Wodonga, Victoria, 3689, Australia. ${ }^{27}$ Faculty of Environmental Science and EULA Chile Centre, Universidad de Concepción, Casilla 160-C, Concepción, Chile. ${ }^{28}$ Institute of Earth Sciences, University of Applied Sciences and Arts of Southern Switzerland, Campus Trevano, 6952 Canobbio, Switzerland. ${ }^{29}$ Australian Rivers Institute, Griffith University, Nathan, Queensland, Australia. ${ }^{30}$ University of Navarra, School of Sciences, Department of Environmental Biology, Irunlarrea 1, 31080-Pamplona, Spain. ${ }^{31}$ Centre for Functional Ecology, Department of Life Sciences, University of Coimbra, Calçada Martim de Freitas, 3000-456 Coimbra, Portugal. ${ }^{32}$ Department of Environmental Science, Policy, and Management, University of California, Berkeley, CA 94720, USA. ${ }^{33}$ LIEC, UMR CNRS 7360, Université de Lorraine, Metz, France. ${ }^{34}$ Centro de Ciências Agrárias e Biológicas, Universidade Estadual Vale do Acaraú, Sobral, CE, Brazil. ${ }^{35}$ Water Research Institute - National Research Council, Italy. ${ }^{36}$ Unidad de Limnología y Recursos Acuáticos (ULRA), Universidad Mayor de San Simón, 
Casilla de Correos 992, Cochabamba, Bolivia. ${ }^{37}$ Department of Ecology and Hydrology, Regional Campus of International Excellence "Campus Mare Nostrum" - University of Murcia, Campus de Espinardo, 30100-Murcia, Spain. ${ }^{38}$ Institute for Applied Ecology, University of Canberra, Bruce, ACT 2601, Australia. ${ }^{39}$ Centre International de Recherche en Agronomie pour le Développement, CIRAD, UPR HORTSYS, F-34398 Montpellier, France. ${ }^{40}$ School of Biological Sciences, University of Canterbury, Christchurch, New Zealand. ${ }^{41}$ INRA, UAR 1275 DEPT EFPA, Centre de recherche de Nancy, Champenoux, France. ${ }^{42}$ School of Marine Sciences, Ruppin Academic Center, 40297 Michmoret, Israel. ${ }^{43}$ Department of Biosciences, Bangalore University, Mangalore 574 199, Karnataka, India. ${ }^{44}$ Department of Ecology and Environmental Science, Umeå University, Umeå, Sweden. ${ }^{45}$ MARE - Marine and Environmental Sciences Centre, Department of Life Sciences, University of Coimbra, 3004-517 Coimbra, Portugal. ${ }^{46}$ Senckenberg Biodiversity and Climate Research Centre (BiK-F), Senckenberganlage 25, 60325 Frankfurt am Main, Germany. ${ }^{47}$ School of Biological and Chemical Sciences, Queen Mary University of London, Mile End Road, London E1 4NS, UK. ${ }^{48}$ Ezemvelo KZN Wildlife, 1 Peter Brown drive, Pietermaritzburg, KwaZulu-Natal, South Africa. ${ }^{49}$ Department of Chemistry, Life Sciences and Environmental Sustainability, University of Parma, Parco Area delle Scienze 11/A - 43124 Parma, Italy. ${ }^{50}$ Department of Aquatic Ecology, Eawag the Swiss Federal Institute of Aquatic Science and Technology, Ueberlandstrasse 133, 8600 Duebendorf, Switzerland ${ }^{51}$ Institute for Ecological Chemistry, Plant Analysis and Stored Product Protection, Julius-Kuehn-Institute, Koenigin-Luise-Str. 19, 14195 Berlin, Germany. ${ }^{52}$ University of Münster, Institute for Evolution and Biodiversity, Department of Limnology, Hüfferstr. 1, 48149 Münster, Germany. ${ }^{53}$ Department of Biology, Faculty of Science, University of Zagreb, Croatia. ${ }^{54}$ Albany Museum, Department of Freshwater Invertebrates, Somerset Street, Grahamstown, 6140, South Africa. ${ }^{55}$ Department of Biology, Universidade de Evora, Evora, Portugal. ${ }^{56}$ Universidad Mayor, Real y Pontificia de San Francisco Xavier de Chuquisaca, Bolivia. ${ }^{57}$ Missouri University of Science and Technology, USA. ${ }^{58}$ Terra Cypria - The Cyprus Conservation Foundation, Cyprus. ${ }^{59}$ Departamento de Ecología y Biología Animal, Universidad de Vigo, 36310-Vigo, Spain. ${ }^{60}$ Department of Botany and Zoology, Faculty of Science, Masaryk University, Brno, Czech Republic. ${ }^{61}$ Department of Biology, University of Montenegro, Cetinjski put b.b., 81000 Podgorica, Montenegro. ${ }^{62}$ Department of Biology and Ecology, Faculty of Sciences and Mathematics, University of Niš, Višegradska 33, 18000 Nis, Serbia. ${ }^{63}$ National Institute of Water and Atmospheric Research, Hamilton, New Zealand. ${ }^{64}$ Laboratoire d'Écologie et Gestion des Ecosystèmes Naturels (LECGEN), University of Tlemcen, 13000 Tlemcen, Algeria. ${ }^{65}$ Israel Nature \& Parks Authority, Israel. ${ }^{66}$ Department of Fish and Wildlife Conservation, Virginia Polytechnic Institute and State University, Blacksburg, VA 24061, USA. ${ }^{67}$ Centre for Tropical Water and Aquatic Ecosystem Research (TropWATER) Freshwater Ecology Research Group College of Science and Engineering, James Cook University, Townsville, 4811, Australia. ${ }^{68}$ Department of Bioscience, Aarhus University, Vejlsøvej, 8600 Silkeborg, Denmark. ${ }^{69}$ Center for Applied Geosciences, Eberhard Karls Universität Tübingen, Tübingen, Germany. *e-mail: Thibault.datry@irstea.fr

\section{Statistics}

Main text: 3000 words

Reference: 37 (and 19 in methods)

2 tables and 3 figures, 10 Supplementary Materials

Keywords: global change, river ecosystem functioning, $\mathrm{CO}_{2}$ emissions, temporary rivers, riparian vegetation, $\mathrm{C}$ cycle

Perennial rivers and streams make a disproportionate contribution to global carbon (C) cycling. However, the contribution of intermittent rivers and ephemeral streams, which 
104 sometimes cease to flow and can dry completely, is largely ignored although they

105 represent over half the global river network. Substantial amounts of terrestrial plant

106 litter accumulate in dry riverbeds and, upon rewetting, this material can undergo rapid

107 microbial processing. We present the results of a global research collaboration which

108 collected and analysed terrestrial plant litter from 212 dry riverbeds spanning major

109 environmental gradients and climate zones. We assessed litter decomposability by

110 quantifying the litter $\mathrm{C}$-to-nitrogen ratio $(\mathrm{C}: \mathrm{N})$ and oxygen $\left(\mathrm{O}_{2}\right)$ consumption in

111 standardised assays and estimated potential short-term $\mathrm{CO}_{2}$ emissions during rewetting

112 events. Aridity, cover of riparian vegetation, channel width, and dry phase duration

113 explained most variability in the quantity and decomposability of plant litter in

114 intermittent rivers and ephemeral streams. Our estimates indicate that a single pulse of

$115 \mathrm{CO}_{2}$ emission upon litter rewetting contribute up to $10 \%$ of daily $\mathrm{CO}_{2}$ emission from

116 perennial rivers and stream, particularly from temperate climates. This implies that the

117 contributions of intermittent rivers and ephemeral streams should be included in global

118 C cycling assessments.

120 Decomposition of terrestrial plant litter is an essential, biosphere-scale ecosystem process ${ }^{1}$. Of

$121120 \mathrm{Pg}$ of organic $\mathrm{C}$ produced by terrestrial plants annually, about half is respired by the

122 plants but only a small fraction is removed by herbivores, so that up to $60 \mathrm{Pg}$ enter the dead

123 organic matter pool ${ }^{1,2}$. Fresh waters make a disproportionate contribution to global C cycling

124 through terrestrial plant litter (TPL) decomposition and atmospheric $\mathrm{CO}_{2}$ emissions ${ }^{3,4}$. This

125 contribution is particularly apparent in perennial rivers and streams, where water and nutrient

126 availability stimulate rapid decomposition by microbes and invertebrate detritivores ${ }^{1,3,5}$. TPL

127 deposited in fresh waters, and the release of its decomposition products, are critical energy

128 sources that support food webs and ecosystem processes, including key $\mathrm{C}$ cycling pathways ${ }^{1,5}$. 
130 A major shortcoming of current estimates of the contribution of rivers and streams to global C

131 cycling ${ }^{3,6,7}$ is the omission of intermittent rivers and ephemeral streams (IRES), in which

132 drying and rewetting events create ecosystems that transition between terrestrial and aquatic

133 phases ${ }^{8,9,10}$. IRES are widespread ecosystems draining a large proportion of terrestrial biomes

134 across all continents and climate types ${ }^{9,12}$. Moreover, IRES are increasing in extent due to

135 global change ${ }^{8,13}$. During the dry phase, TPL deposited on the riverbed accumulates,

136 decomposing only slowly through photodegradation and terrestrial decomposer activity ${ }^{14,15}$.

137 Then, when flow resumes, the accumulated material is mobilised and transported

138 downstream ${ }^{16,17}$ (Supplementary Material 1). Concentrations of particulate and dissolved

139 organic matter in advancing wetted fronts exceed baseflow concentrations by several orders

140 of magnitude ${ }^{16}$. IRES have therefore been conceptualised as punctuated biogeochemical

141 reactors 9 .

142

143 To understand the role of IRES in global C cycling, global-scale data are needed to

144 characterise the variables controlling TPL accumulation in dry channels and its

145 decomposability upon flow resumption. Climate influences the type and productivity of

146 riparian vegetation ${ }^{18}$ and the flow regimes of IRES ${ }^{8,13}$. Channel topography and flow

147 conditions, including the timing and duration of dry periods ${ }^{14}$, control TPL deposition and

148 retention, with wide channels receiving proportionally less riparian material than narrow

149 ones $^{19}$. TPL decomposability is typically altered during dry phases, due to partial degradation

150 or leaching of labile constituents during rainfall events, relative accumulation of recalcitrant

151 compounds, and leaching of labile constituents, relative accumulation of recalcitrant

152 compounds, and impoverishment of nutrients in terrestrial conditions ${ }^{15,20}$. Therefore, we

153 predict that TPL accumulation and decomposability would be a function of climate, riparian 
154 vegetation, channel topography, and duration of the dry phase (Fig. 1). We explored these

155 relationships by assessing the quantity and decomposability of accumulated TPL in 212 dry

156 river channels located in 22 countries distributed across wide environmental gradients and

157 multiple climate zones $^{8}$ (Supplementary Material 2).

Terrestrial plant litter accumulation in dry riverbeds

160 Our results refine current understanding of the global distribution and variability in TPL

161 accumulation in IRES during dry phases. The quantity of TPL collected in 212 dry riverbeds

162 (Supplementary Material 2) ranged from 0 to $8291 \mathrm{~g}$ dry mass $\mathrm{m}^{-2}$ (mean \pm S.D. $=277 \pm$

163 796, median $=102 \mathrm{~g} \mathrm{~m}^{-2}$; Table 1). This material mainly comprised leaf litter (LL) and wood

164 (41\% and 39\% of the total mass, respectively), whereas herbs, fruits and catkins accounted for

$165<20 \%$ of the total mass (Table 1). The quantity of LL ranged from 0-963 $\mathrm{g} \mathrm{m}^{-2}$ (mean \pm S.D.

$166=88 \pm 139$, median $\left.=36 \mathrm{~g} \mathrm{~m}^{-2}\right)$.

167

168 Relationships between TPL quantity and environmental variables were assessed using

169 Random Forest models (RF), which are highly flexible regression techniques suitable for

170 modelling responses that show complex relationships with environmental conditions (e.g.,

171 climate, riparian zone, flow regime, channel topography). RF based on data from all samples

172 explained $41.4 \%$ and $38.3 \%$ of the total variance in TPL and LL quantity, respectively (Table

173 2, Fig. 2). Supporting our conceptual model (Fig. 1), aridity, mean annual precipitation,

174 catchment area, and dry period duration were the most important predictors of TPL quantity

175 (Table 2). Aridity, river width, riparian cover, time since senescence, and dry period duration

176 were most influential to determine LL accumulation (Table 2). LL quantity generally

177 increased with riparian cover and decreased with river width (Fig. 2). Relationships with time

178 since senescence, aridity, and dry period duration were more complex. LL quantity decreased 
as the aridity index increased to 250 , increased sharply until it reached 650 and then plateaued

180 (Fig. 2). LL quantity also increased almost linearly as dry period duration increased to $200 \mathrm{~d}$,

181 and then dropped sharply (Fig. 2). The quantity of LL fell for 320 days after estimated

182 senescence and then rose slightly (Fig. 2).

183 The greatest quantity of terrestrial material, in particular LL, was reported from first-order,

184 forested, temperate IRES, suggesting these sites are hotspots of organic matter accumulation

185 in dendritic river networks. This finding concurs with patterns predicted by the River

186 Continuum Concept $(\mathrm{RCC})^{21}$ but differ from its predictions regarding the fate of TPL entering

187 river channels. According to the RCC, a large portion of TPL entering forested headwaters is

188 immediately processed by heterotrophic microbes and invertebrate shredders, generating

189 significant amounts of fine-particulate organic matter that is exported downstream. In

190 contrast, we found TPL accumulations in dry channels to be greatly increased compared to

191 perennial rivers ${ }^{8,14}$, because the absence of flowing water limits biological activity and

192 physical abrasion. During the initial phases when flow resumes, much of this material can

193 then be transported and further processed downstream ${ }^{9,10,16}$.

194

195 Overall, LL accumulation in IRES matches global patterns in terrestrial inputs ${ }^{1,20}$, revealing

196 strong biogeochemical and ecological links between rivers and adjacent terrestrial

197 ecosystems. The positive relationship between the degree of aridity and the quantity of

198 accumulated LL probably reflects water-limited riparian plant growth ${ }^{22}$, while the saturating

199 relationship observed above an index value of 700 suggest that, in humid conditions, LL

200 accumulation becomes limited by other factors. LL quantities in dry channels reflect a balance

201 between riparian and upstream inputs, and losses due to dry-phase decomposition and

202 downstream export during phases of flow. Downstream effects of LL transport and processing 
when flow resumes will also depend on the decomposability of the accumulated organic

204 matter.

\section{Decomposability of accumulated leaf litter}

The mass C:N ratio of LL, as a first proxy of decomposability, ranged from 17 to 154 (mean \pm S.D. $=46 \pm 23$ ) and was driven by climate, riparian cover, and dry period duration, as predicted by our conceptual model (Fig. 1). However, the RF model explained only $14.9 \%$ of the total variance in $\mathrm{C}: \mathrm{N}$ (Table 2). The relationship of the C:N ratio with mean annual potential evapotranspiration (PET) was not monotonic in that the $\mathrm{C}: \mathrm{N}$ ratio increased sharply between about 700 and $900 \mathrm{~mm} \mathrm{PET} \mathrm{year}^{-1}$ and then gradually decreased (Supplementary Material 3). The C: $\mathrm{N}$ ratio decreased with riparian cover and the aridity index, the latter

214 relationship resembling the reverse of its response to dry period duration (Supplementary

215 Material 3). Aridity was an important influence on C:N, with lower ratios reported for low216 aridity environments, including tropical conditions, compared to other climate types ${ }^{20,23}$.

217 More research is needed to determine how plant species richness, vegetation structure and

218 functional diversity in riparian zones affect the C:N and decomposability of LL in dry 219 riverbeds.

221 Decomposability was also related to preconditioning after LL deposition on dry riverbeds. A

222 few days of drying on the riverbed decreased the C:N ratio of LL, whereas longer drying 223 periods resulted in increases, with peaks occurring after 100 days before $\mathrm{C}: \mathrm{N}$ declined again, 224 levelling off after 200 days (Supplementary Material 3). The increase in C:N with dry 225 period duration suggests that nutrients, along with other soluble compounds, are preferentially 226 leached from LL in dry riverbeds, resulting in litter composed mostly of nutrient-poor 227 structural compounds such as cellulose and lignin ${ }^{24}$. The initial decomposability of LL falling 
onto dry riverbeds and subsequent quality changes affect decomposition in both the receiving and downstream reaches ${ }^{16}$. Thus, climate change-related extensions of dry periods ${ }^{13}$ could increase downstream transport of low-quality LL, with potential repercussions on detrital food webs and associated ecosystem functions and services.

\section{Respiration and $\mathrm{CO}_{2}$ release after leaf litter rewetting}

We did not determine decomposition rates directly, but used a proxy of terrestrial litter decomposability by measuring oxygen consumption related to rewetting in laboratory conditions. Oxygen consumption rates of rewetted LL ranged from 0.004 to $0.97 \mathrm{mg} \mathrm{O}_{2} \mathrm{~g}^{-1}$ dry mass $^{-1}($ mean \pm S.D. $=0.36 \pm 0.20$, median $=0.29)$. These values are in the upper range of respiration rates reported from coarse-particulate organic matter in fresh waters and soils (0.009-0.55 and $<0.001-0.35 \mathrm{mg} \mathrm{O}_{2} \mathrm{~g}^{-1}$ dry mass $\mathrm{h}^{-1}$ for fresh waters and soils, respectively; Supplementary Material 4). This indicates that rewetting events are associated with intense biological activity, when the highly labile $\mathrm{C}$ fuelling the initial respiration after rewetting can be rapidly metabolised by most heterotrophic microorganisms present in the litter ${ }^{14}$. The global RF model explained $36.8 \%$ of the total variation in $\mathrm{O}_{2}$ consumption rates, with the most important predictors being the riparian forest proportion in the catchment, catchment area, the time since senescence, dry period duration, aridity, and the C:N ratio (Table 2, Supplementary Material 5). Rates increased with catchment area, and decreased with forest proportion, aridity, $\mathrm{C}: \mathrm{N}$, time since senescence, and dry period duration. Upon flow resumption, higher microbial respiration rates are triggered when previous drying events are short compared to extended dry phases. The predicted increase in the frequency of drying events $^{9,13}$ might have strong implications on IRES metabolism and thus increase their contribution to the global $\mathrm{C}$ cycle through $\mathrm{CO}_{2}$ emissions upon rewetting. 
253 Our estimates of $\mathrm{CO}_{2}$ emissions from IRES upon LL rewetting ranged from 0 to $13.7 \mathrm{~g} \mathrm{CO}_{2}$

$254 \mathrm{~m}^{-2} \mathrm{day}^{-1}($ mean \pm S.D. $=0.88 \pm 1.51$, median $=0.42)$, which is in the upper range of

255 previously reported daily emission rates from fresh waters and soils (Supplementary

256 Material 6). Notably, the highest daily values are 10-fold higher than those reported in the

257 most comprehensive estimates of $\mathrm{CO}_{2}$ emission rates available from inland waters ${ }^{3}$, in which

258 reservoirs are expected to release up to $0.34 \mathrm{~g} \mathrm{CO}_{2} \mathrm{~m}^{-2}$ day $^{-1}$ and perennial streams up to 1.75

$259 \mathrm{~g} \mathrm{CO}_{2} \mathrm{~m}^{-2}$ day $^{-1}$. Our highest potential $\mathrm{CO}_{2}$ emission rate associated with LL rewetting could

260 thus represent up to $152 \%$ of previous estimates from perennial streams and rivers when

261 comparing daily emission rates $(\min =0 \%$, mean $=3-10 \%, \max =47-152 \%$; Supplementary

262 Material 7a). This is remarkable, especially since our estimates are conservative, because

263 they are mainly based on microbial activity on LL and exclude sediment respiration. The

264 highest emission rates were found at sites characterised neither by the highest $\mathrm{O}_{2}$ consumption

265 rates nor by the highest quantities of accumulated LL, indicating that the two variables are

266 uncorrelated. This highlights the need to consider both LL quantity and decomposability, to

267 evaluate the role of IRES in the global C cycle.

269 The RF model explained $34.9 \%$ of the total variation in the potential $\mathrm{CO}_{2}$ released with

270 estimated time since senescence, aridity, and drying duration as the most important predictors

271 (Table 2, Fig. 3a). Relationships were typically non-monotonic. The $\mathrm{CO}_{2}$ released decreased

272 sharply until 85 days after estimated senescence, before remaining relatively low and stable

273 (Fig. 3a). $\mathrm{CO}_{2}$ release decreased till an aridity index value of 230, then increased sharply till

274700 to decrease again and stabilise at values above 800 (Fig. 3a). Last, rates of $\mathrm{CO}_{2}$ release

275 remained stable for $200 \mathrm{~d}$ of dry riverbeds, but sharply decreased thereafter (Fig. 3a).

276 Although IRES release $\mathrm{CO}_{2}$ during both flowing ${ }^{3,25}$ and dry ${ }^{26}$ phases, our study suggests that

277 early stages of rewetting can be considered hot moments ${ }^{9,11}$ or control points ${ }^{27}$ of $\mathrm{CO}_{2}$ release. 
This finding is important because global estimates of $\mathrm{CO}_{2}$ release focusing on perennial rivers ${ }^{3,4,7,25}$ have missed emissions from at least $84,000 \mathrm{~km}^{2}$ of river channel areas (representing $\sim 12.3 \%$ of total river and stream areas) by overlooking IRES $^{3,28}$.

\section{Differences among climate zones}

Our global study demonstrates that the quantities of organic material accumulating during dry phases in riverbeds vary substantially among climate zones. Temperate IRES accumulated more LL $\left(\right.$ mean \pm S.D. $=97 \pm 152$, median $=41$ g dry mass $\left.\mathrm{m}^{-2}\right)$ than those in the tropics $\left(\right.$ mean \pm S.D. $=32 \pm 44$, median $=9$ g dry mass $\left.\mathrm{m}^{-2}\right)$ and arid climates $($ mean \pm S.D. $=45 \pm$ 64, median $=7 \mathrm{~g}$ dry mass $\left.\mathrm{m}^{-2}\right)($ ANOVA, $\mathrm{P}<0.001)$. Of the sampled riverbeds, $150,31,19$, and 10 were located in temperate, arid, tropical and continental climates, respectively, reflecting the geographical spread of current IRES research ${ }^{29}$ and highlighting that our results need to be interpreted with caution in less well-represented climate classes, particularly in alpine (only a single location), continental and, to a lesser extent, tropical IRES. When run separately for different climate zones, RF model performance to predict the quantity of accumulated LL was indeed much higher for temperate and arid $(36.1 \%$ and $26.8 \%$ of total variance explained, respectively) than for tropical (5.6\%) climates. Thus, our conclusions are more solid in temperate and arid climates, where IRES are widespread, compared to the tropics $^{30,31}$. For example, IRES represent up to $45 \%$ of the hydrological network in temperate France $^{32}$ and up to $96 \%$ in the arid south-western USA ${ }^{33,34}$. Tropical IRES often have higher annual LL inputs than temperate forests ${ }^{35}$, but our ability to predict their LL accumulation in these riverbeds was reduced, probably because of often continuous leaf fall ${ }^{36}$. This result might indicate that $\mathrm{C}$ cycling in IRES is less punctuated in tropical than in other climates, although identical predictors were retained by the respective RF models, indicating that litter accumulation is controlled by common factors across all climatic zones. 
304 Our findings on LL accumulation were paralleled by estimates of $\mathrm{CO}_{2}$ release upon rewetting,

305 which were also much higher in temperate (mean \pm S.D. $=1.06 \pm 1.76 \mathrm{~g} \mathrm{CO}_{2} \mathrm{~m}^{-2}$ ) than in arid 306 and tropical IRES $\left(0.48 \pm 0.68\right.$ and $0.28 \pm 0.35 \mathrm{~g} \mathrm{CO}_{2} \mathrm{~m}^{-2}$, respectively). However, this

307 comparison is influenced by the limited ability of our models to predict $\mathrm{CO}_{2}$ release from arid 308 IRES (4.4\% of the variance explained) compared to temperate and tropical IRES (33.5 and $30916.8 \%$ of the variance explained, respectively). This may reflect the role of abiotic processes 310 such as photodegradation for LL decomposition in water-limited river ecosystems ${ }^{15}$ or the 311 influence of plant functional traits, not included in our model, that are involved in the 312 protection from desiccation and solar radiation, such as the quantities of waxes and phenolic 313 compounds ${ }^{37}$.

\section{Implications and perspectives}

316 Our global study spanning 212 reaches on all continents (i) enabled us to document the extent 317 of global variation in TPL and LL quantity and quality across dry riverbeds, and (ii) revealed 318 high $\mathrm{O}_{2}$ consumption and $\mathrm{CO}_{2}$ release rates after LL rewetting, notably in temperate regions. 319 These findings support the notion of IRES as punctuated biogeochemical reactors ${ }^{9}$, characterised by distinct phases of $\mathrm{C}$ accumulation and processing with much higher temporal variability in process rates than in perennial river ecosystems. Transport distance and site of

322 litter deposition and processing after flow resumes will vary with river morphology and the 323 magnitude of the flow pulse ${ }^{16}$. However, except during extreme flow conditions, much of the 324 mobilised litter will remain in river channels and riparian areas, where it decomposes at rates 325 similar to those in perennial rivers. Since these rates are much faster than in upland terrestrial 326 sites $^{1,14}$, these findings suggest that neglecting IRES leads to a notable underestimation of the 327 contribution of the world's river network to the total global $\mathrm{CO}_{2}$ flux to the atmosphere. Our 
study suggests that in addition to globally relevant amounts of $\mathrm{CO}_{2}$ released from IRES

329 during both $\operatorname{dry}^{26}$ (Supplementary Material 7b) and flowing phases, rewetting events act as

330 control points ${ }^{27}$. This would imply upward revision of organic matter transformations and

$331 \mathrm{CO}_{2}$ emissions from river networks on the global scale. Indeed, based on the comparison of

332 daily $\mathrm{CO}_{2}$ emission rates with those reported from perennial rivers and streams, IRES could

333 increase estimates of global $\mathrm{CO}_{2}$ emissions from streams and rivers by $7-152 \%$, the $\mathrm{CO}_{2}$

334 released from LL during a single rewetting event alone contributing roughly from 3 to $10 \%$ of

335 this increase (Supplementary Material 7a). Likewise, taking IRES into account would

336 improve estimates of the consequences of global climate change on $\mathrm{C}$ cycling, given that the

337 spatial extent of IRES will increase, and period of drying will become more prolonged, in

338 many regions $9,11,13$.

340 The data and conceptual framework presented here provide the basis needed to develop

341 models of litter decomposition and C cycling in fresh waters that include IRES. The next

342 steps would be to quantify $\mathrm{CO}_{2}$ emissions upon flow resumption in situ ${ }^{16}$ and collect data on

343 LL quantity and decomposability for continental and other climates that are not well

344 represented at present. $\mathrm{CO}_{2}$ emissions from dry phases, suggested recently to be substantial ${ }^{26}$,

345 along with those from flowing phases $^{3}$, need to be integrated with those during wetting

346 events, and temporal variability (including its dependency on other environmental conditions,

347 such as temperature) be studied for extended periods after flow resumes to build adequate

348 quantitative models of global C cycling that consider the spatio-temporal dynamics of IRES

349 under present and future climatic conditions.

$351 \quad$ References

352 1. Boyero, L. et al. A global experiment suggests climate warming will not accelerate litter decomposition in 353 streams but might reduce carbon sequestration. Ecol. Lett. 14, 289-294 (2011). 
2. Beer, C. et al. Terrestrial gross carbon dioxide uptake: global distribution and covariation with climate. Science 329, 834-838 (2010).

356 3. Raymond, P. A. et al. Global carbon dioxide emissions from inland waters. Nature 503, 355-359 (2013).

357 4. Hotchkiss, E. R. et al. Sources of and processes controlling $\mathrm{CO}_{2}$ emissions change with the size of streams and $358 \quad$ rivers. Nat. Geosci. 8, 696-699 (2015).

359 5. Gessner, M. O. et al. Diversity meets decomposition. Trends Ecol. Evol. 25, 372-380 (2010).

360 6. Battin, T. J. et al. The boundless carbon cycle. Nature 2, 598-600 (2009).

361 7. Butman, D. et al. Aquatic carbon cycling in the conterminous United States and implications for terrestrial 362 carbon accounting. Proc. Natl. Acad. Sci. USA 113, 58-63 (2016).

363 8. Datry, T., Corti, R., Foulquier, A., Von Schiller, D. \& Tockner, T. One for all, all for one: a global river 364 research network. Eos 97, 13-15 (2016).

365 9. Larned, S. T., Datry, T., Arscott, D. B. \& Tockner, K. Emerging concepts in temporary- river ecology. $366 \quad$ Freshwater Biol. 55, 717-738 (2010).

367 10. Stanley, E. H., Fisher, S. G. \& Grimm, N. B. Ecosystem expansion and contraction in streams. BioScience 368 47, 427-435 (1997).

369 11. Datry, T., Larned, S. T. \& Tockner, K. Intermittent rivers: a challenge for freshwater ecology. BioScience 64, $370 \quad 229-235$ (2014).

371 12. Acuña, V. et al. Why should we care about temporary waterways? Science 343, 1080-1081 (2014).

372 13. Jaeger, K. L., Olden, J. D. \& Pelland, N. A. Climate change poised to threaten hydrologic connectivity and 373 endemic fishes in dryland streams. Proc. Natl. Acad. Sci. USA 111, 13894-13899 (2014).

374 14. Foulquier, A., Artigas, J., Pesce, S. \& Datry, T. Drying responses of microbial litter decomposition and 375 associated fungal and bacterial communities are not affected by emersion frequency. Freshw. Sci. 34, 1233$3761244(2015)$.

377 15. Austin, A. T. \& Vivanco, L. Plant litter decomposition in a semi-arid ecosystem controlled by 378 photodegradation. Nature 442, 555-558 (2006).

379 16. Corti, R. \& Datry, T. Invertebrates and sestonic matter in an advancing wetted front travelling down a dry $380 \quad$ river bed (Albarine, France). Freshw. Sci. 31, 1187-1201 (2012).

381 17. Rosado, J., Morais, M. \& Tockner, K. Mass dispersal of terrestrial organisms during first flush events in a 382 temporary stream. River Res. Appl. 31, 912-917 (2015). 
18. Michaletz, S. T., Cheng, D., Kerkhoff, A. J. \& Enquist, B. J. Convergence of terrestrial plant production across global climate gradients. Nature 512, 39-43 (2014).

19. Ehrman, T. P. \& Lamberti, G. A. Hydraulic and particulate matter retention in a 3rd-order Indiana stream. J. N. Am. Benthol. Soc. 11, 341-349 (1992).

20. Boyero, L. et al. Riparian plant litter quality increases with latitude. Sci. Rep. 7, 10562 (2017).

21. Vannote, R. L., Minshall, G. W., Cummins, K. W., Sedell, J. R. \& Cushing, C. E. The River Continuum Concept. Can. J. Fish. Aquat. Sci. 37, 130-137 (1980).

22. Olson, J. S. Energy storage and the balance of producers and decomposers in ecological systems. Ecology $\mathbf{4 4}$, 322-331 (1963).

23. Aerts, R. Climate, leaf litter chemistry and leaf litter decomposition in terrestrial ecosystems: a triangular relationship. Oikos 79, 439-449 (1997).

24. Cleveland, C. C., Neff, J. C., Townsend, A. R. \& Hood, E. Composition, dynamics, and fate of leached dissolved organic matter in terrestrial ecosystems: results from a decomposition experiment. Ecosystems 7, 175-285 (2004).

25. Hasler, C. T., Butman, D., Jeffrey, J. D. \& Suski, C. D. Freshwater biota and rising $\mathrm{pCO}_{2}$ ? Ecol. Lett. 19, 98108 (2016).

26. Gómez-Gener, L. et al. When water vanishes: magnitude and regulation of carbon dioxide emissions from dry temporary streams. Ecosystems $\mathbf{1 9}, 710-723$ (2016).

27. Bernhardt, E. S. et al. Control points in ecosystems: moving beyond the hot spot hot moment concept.

403 28. Benstead, J. P. \& Leigh, D. S. An expanded role for river networks. Nat. Geosci. 5, 678-679 (2012).

404 29. Leigh, C. et al. Ecological research and management of intermittent rivers: an historical review and future 405 directions. Freshwater Biol. 61, 1181-1199 (2016).

406 30. Stubbington, R., England, J., Wood, P. J. \& Sefton, C. E. Temporary streams in temperate zones: 407 recognizing, monitoring and restoring transitional aquatic- terrestrial ecosystems. WIRES Water 4, e1223 $408 \quad$ (2017).

409 31. Datry, T., Bonada, N. \& Boulton, A. J. Introduction. In Intermittent Rivers and Ephemeral Streams: Ecology 410 and Management (eds. Datry, T., Bonada, N. \& Boulton, A. J.) 1-20 (Academic Press, 2017).

411 32. Snelder, T. H. et al. Regionalization of patterns of flow intermittence from gauging station records. Hydrol. Earth Syst. Sci. 17, 2685-2699 (2013). 

(2000).

34. Levick, L. R. et al. The Ecological and Hydrological Significance of Ephemeral and Intermittent Streams in the Arid and Semi-arid American Southwest (U.S. Environmental Protection Agency, 2008).

35. Huston, M. A. \& Wolverton, S. The global distribution of net primary production: resolving the paradox. Ecol. Monogr. 79, 343-377 (2009).

36. Murphy, P. G. \& Lugo, A. E. Ecology of tropical dry forest. Ann. Rev. Ecol. Syst. 17, 67-88 (1986).

37. De Deyn, G. B., Cornelissen, J. H. C. \& Bardgett, R. D. Plant functional traits and soil carbon sequestration in contrasting biomes. Ecol. Lett. 11, 516-531 (2008).

\section{Acknowledgements}

This study was made possible thanks to a large collective effort of a global research network entitled the "1000 Intermittent River Project" (1000IRP) merging individual knowledge, forces and passion through simple, consistent and comparable joint field experiments worldwide.

\section{Author contributions}

T. Datry, A. Foulquier, R. Corti, D. von Schiller, and K. Tockner assumed responsibility for the overall project planning and coordination. All authors collected plant litter in their countries and processed and analysed this material. The centralised lab analyses were conducted by T. Datry, A. Foulquier, R.Corti, C. Mendoza-Lera, and J.C. Clement. The data compilation and database management was carried out by R. Corti and C. Mendoza-Lera. The data analyses were performed by T. Datry, R.Corti, A. Foulquier, and C. Mendoza-Lera. T. Datry led the writing of the manuscript with A. Foulquier and notable contributions by M.O.

437 Gessner, B. Gücker, M. Moléon and R. Stubbington. All other authors commented on and 438 contributed to revising draft versions. 
440 Corresponding author: Correspondence and request for material should be addressed to Dr.

441 Thibault Datry, IRSTEA Lyon, France. thibault.datry@irstea.fr

443 Competing interests

444 The authors declare no competing financial or non-financial interests.

Table and Figure captions

Table 1: Quantity (g dry mass. $\mathrm{m}^{-2}$ ) of terrestrial plant litter collected in dry riverbeds (Min: minimum, Max: maximum, Mean, S.D.: standard deviation, Fraction: \% of the total quantity.

Table 2. Detailed results of global Random Forest (RF) models on five response variables. The variables used as predictors are described in Supplementary Material 8. INC MSE corresponds to the increase in the mean squared error of the predictions after permutation. INC Node Purity is the average decrease in node impurity measured as residual sum of squares. Both are used to assess the importance of predictors in an RF model. The higher the value of both measures, the more important the variable.

Figure 1. Main variables predicted to control plant litter accumulation and

461 terrestrial plant material is a function of the input of litter from riparian vegetation mediated 462 by its retention that depends on channel topography and the duration of dry events. Channel 463 topography and composition of the riparian vegetation are driven by flow regimes and, 464 ultimately, climate. Climate also influences the condition of the litter accumulated during dry 
phases and hence its preconditioning. Photo credits: D. von Schiller (left panel) and M.

466 Moléon (right panel).

Figure 2. Partial dependence of the probability of the quantity of leaf litter (LL)

accumulated in dry reaches. Variables are shown from the top left to the bottom right in

order of decreasing importance. The plots show the marginal contribution to probability of the quantity of LL accumulated in dry reaches (marginal response, y-axis) as a function of the predictors (i.e. when the other contributing predictors are held at their mean). The rug plots on the horizontal axes show deciles of the predictors.

Figure 3. a. Partial dependence of the probability of the $\mathrm{CO}_{2}$ released by rewetted leaf

litter (LL) over $24 \mathbf{h}$. Variables are shown from left to right in order of decreasing importance. The plots show the marginal contribution to probability of the $\mathrm{CO}_{2}$ released by rewetted LL over $24 \mathrm{~h}$ (marginal response, $\mathrm{y}$-axis) as a function of the predictors (i.e. when the other contributing predictors are held at their mean). The rug plots on the horizontal axes show deciles of the predictors. b. potential $\mathrm{CO}_{2}$ released mapped onto the original sampling reaches.

\section{Methods}

Sampling design. Terrestrial plant litter (TPL) deposited on dry riverbeds was collected by participants of an international consortium (http://1000_intermittent_rivers_project.irstea.fr ${ }^{8}$ ) following a standardised protocol. In total, 212 near-natural river reaches were studied in 22 countries spanning 13 Köppen-Geiger climate classes (Supplementary Material 2). Briefly, the sampled river reaches were $10 \times$ the average active channel widths to cover a representative area of each river channel and to ensure consistent sampling effort across reaches ${ }^{38}$. The active channel was defined as the area of frequently inundated and exposed 
riverbed sediments between established edges of perennial, terrestrial vegetation and/or abrupt changes in slope $\mathrm{e}^{39}$. TPL was collected by hand from $1 \mathrm{~m}^{2}$ quadrats placed randomly within each reach during a dry phase. The quadrats covered $\sim 5 \%$ of the reach surface area (e.g. five quadrats in a $100 \mathrm{~m}^{2}$ reach). Different types of TPL (i.e. leaves, wood, fruits, catkins, herbs) were stored in separate airtight plastic bags.

\section{Environmental variables. A set of 22 environmental variables reflecting reach} characteristics at different spatial scales was estimated or calculated for each site (Supplementary Material 8). Seventeen variables were determined locally. Mean annual temperature and precipitation were extracted from the WorldClim.org database, which gives 1-km spatial resolution climate surfaces for global land areas over the period 1970-2000.

Mean annual potential evapotranspiration (PET) and mean annual aridity were determined using the Global Aridity and PET database published by the Consortium for Spatial Information (CGIARCSI, http://www.cgiar-csi.org) using the WorldClim.org database. PET is a measure of the ability of the atmosphere to remove water through evapotranspiration and was calculated as a function of annual mean temperature, daily temperature range and extraterrestrial radiation between 1950 and 2000. Mean annual aridity was assessed using an aridity index ${ }^{40}$ and expressed as $1000 \times$ precipitation / PET between 1950 and 2000. Aridity index values were high in humid and low in arid conditions. Climate zones following the Köppen-Geiger system were determined from the global climate map derived from long-term monthly precipitation and temperature time series in a grid of weather stations and interpolated among stations using a two-dimensional (latitude and longitude) thin-plate spline with tension onto a $0.1^{\circ}$ by $0.1^{\circ}$ grid for each continent ${ }^{41}$. Last, we estimated time since leaf abscission as the time between the estimated onset of leaf senescence and the sampling date. Although leaf fall is more continuous in tropical areas than in other climate zones, to facilitate 
516 comparison among sites, onset of leaf senescence was set to the $1^{\text {st }}$ of September and the $15^{\text {th }}$

517 of February in the northern and southern hemispheres, respectively ${ }^{42}$.

519 Litter drying, weighing and grinding. TPL was transported to local laboratories within $8 \mathrm{~h}$

520 of collection when possible and oven dried at $60{ }^{\circ} \mathrm{C}$ for $\geq 12 \mathrm{~h}(<24 \mathrm{~h}$ for leaves). Fresh

521 material such as fruits or wood was dried at room temperature for 1 week before oven drying.

522 The dried material was weighed to the nearest gram. Although wood can account for

523 considerable volumes of TPL deposited in riverbeds, it is far more recalcitrant than leaf litter

524 (LL). Therefore, we focused on LL in our assessment of TPL decomposability during short-

525 term rewetting events. LL was thoroughly mixed before taking a 60-g subsample that was first

526 shredded by hand and passed through a $0.5-\mathrm{cm}$ mesh screen, then shipped to the IRSTEA

527 laboratory (Lyon, France) for further processing.

529 Decomposability of leaf litter. Laboratory measurements can provide a useful means to

530 address global-scale environmental research questions ${ }^{43}$ and overcome the current data

531 shortage on intermittent rivers and ephemeral streams. In particular, they facilitate tests of

532 between-reach variability in $\mathrm{O}_{2}$ consumption rates in a standardised way and identification of

533 the primary drivers responsible for the observed variability. Although we did not quantify

534 decomposition rates directly, we assessed two proxies of LL decomposability, the C:N mass

535 ratio and oxygen $\left(\mathrm{O}_{2}\right)$ consumption rate after rewetting.

537 Three 10-mg LL subsamples were taken from each sample, ground to $5 \mu \mathrm{m}$ with a ball mill 538 (MM301, Retsch GmbH, Haan, Germany) and the C:N ratio determined with an elemental 539 analyzer (FlashEA 1112, Fisher Scientific, Waltham, Massachusetts, USA). O 2 consumption 540 was determined in respiration flasks placed in a climatic room at $20{ }^{\circ} \mathrm{C}$. LL subsamples were 
541 processed in 10 successive batches of 25-50 subsamples. Each batch was incubated in three

542 200-L polyethylene containers filled with tap water at room temperature to prevent $\mathrm{O}_{2}$

543 exchange with the atmosphere. For each subsample, two analytical replicates were processed

544 by placing $0.1 \mathrm{~g} \mathrm{LL}$ into 250 -mL glass respiration flasks filled with Volvic ${ }^{\circledR}$ mineral water,

545 then sealed airtight using a 3.2-mm-thick silicon-PTFE septum and a cut-out open-top cap.

546 Care was taken to ensure air bubbles were excluded. $\mathrm{O}_{2}$ concentrations were measured with a

547 needle-based micro-optode (Oxygen Microsensor PM-PSt7; PreSens, Regensburg, Germany)

548 using a stand-alone, portable, fiber-optic $\mathrm{O}_{2}$ meter (Microx 4 trace; PreSens, Regensburg,

549 Germany). Incubations were run for approximately $24 \mathrm{~h}$ (range of incubation times: 23.4-25.8

$550 \mathrm{~h}$; mean \pm S.D. $=24.3 \pm 2.0 \mathrm{~h}$ ) to simulate short-term rewetting events. We used LL

551 communities as a source of microbes, because dry LL hosts dormant communities that can

552 quickly resume activity after litter rewetting ${ }^{44}$. We also ran tests to ensure our oxygen

553 consumption rates were realistic. This was achieved by using LL, different sources of water

554 with and without a standard inoculum from local streams (see below).

$556 \mathrm{O}_{2}$ concentrations were measured twice, $2 \mathrm{~h}$ and $24 \mathrm{~h}$ after the respiration flasks were filled 557 with water. We waited for $2 \mathrm{~h}$ before taking the first measurement to allow gas release from 558 air-saturated pores within the $\mathrm{LL}^{45}$. Although the respiration flasks were carefully filled 559 without bubbling the water, we left them open for $2 \mathrm{~h}$ while the LL released gas, to ensure 560 that $\mathrm{O}_{2}$ concentration was saturated, but not supersaturated to avoid a notable underestimation 561 of respiration rates over 24 h. Flasks were gently agitated every $6 \mathrm{~h}$ during the incubation 562 period and before each measurement to ensure homogenous $\mathrm{O}_{2}$ concentrations in the water. 563 For each batch, $\mathrm{O}_{2}$ concentrations were also measured in three control respiration flasks filled 564 with Volvic ${ }^{\circledR}$ mineral water only. Microbial respiration associated with LL (R: $\mathrm{mg} \mathrm{O}_{2} \mathrm{~g}^{-1} \mathrm{LL}$ 565 dry mass $\mathrm{h}^{-1}$ ) was calculated as: 


$$
R=\frac{\frac{\left(O_{2 \text { sample }}^{2 h}-O_{2 \text { sample }}^{24 h}\right)-\left(O_{2 \text { control }}^{2 h}-O_{2 \text { control }}^{24 h}\right)}{\text { incubation time }(h)} \times \text { respiration flask volume }}{(g)}
$$

where $\mathrm{O}_{2}$ is the dissolved $\mathrm{O}_{2}$ concentration $\left(\mathrm{mg} \mathrm{L}^{-1}\right)$; the subscripts sample and control refer to

each analytical replicate and the mean $\mathrm{O}_{2}$ of the three control respiration flasks; and the

superscripts $2 \mathrm{~h}$ and $24 \mathrm{~h}$ correspond to the $\mathrm{O}_{2}$ concentrations measured $2 \mathrm{~h}$ and $24 \mathrm{~h}$ after the

flask was filled, respectively. $R$ was then standardised to $20{ }^{\circ} \mathrm{C}$ to correct for small (i.e., \pm

$571 \quad 1.1^{\circ} \mathrm{C}$ ) temperature variations during the measurements, assuming that $\mathrm{O}_{2}$ consumption rates

572 double with a temperature increase of $10{ }^{\circ} \mathrm{C}^{46}$. The mean of the two analytical replicates was used as a measure of microbial respiration associated with LL rewetting for each sample. For 10 samples, we had not sufficient litter material to conduct the respiration measures and for another 6 , the material was not adequately processed by the collectors and was thus excluded from the analysis. Hence, the total number of samples analysed for $\mathrm{O}_{2}$ consumption rates was 196 (Supplementary Material 9).

The total potential $\mathrm{CO}_{2}$ released per $\mathrm{m}^{2}$ of riverbed over $24 \mathrm{~h}$ after rewetting was estimated by multiplying, for each sampling site, the amount of accumulated LL (in $\mathrm{g}$ per $\mathrm{m}^{2}$ ) by the rate of

$581 \mathrm{O}_{2}$ consumption ( $\mathrm{mg} \mathrm{O}_{2} \mathrm{~g}^{-1} \mathrm{LL}$ dry mass $\mathrm{h}^{-1}$ ) over $24 \mathrm{~h}$ (Supplementary Material 9). The

582 obtained estimates of $\mathrm{O}_{2}$ consumption $\left(\mathrm{mg} \mathrm{O}_{2} \mathrm{~m}^{-2}\right.$ day $\left.{ }^{-1}\right)$ were then converted into $\mathrm{CO}_{2}$

583 production $\left(\mathrm{mg} \mathrm{CO}_{2} \mathrm{~m}^{-2} \mathrm{day}^{-1}\right)$ by assuming a respiratory quotient of $1^{47}$.

Sensitivity of $\mathrm{O}_{2}$ consumption measurements. To explore the sensitivity of our laboratory

586 protocol to assess LL respiration in the initial stage of rewetting, we compared $\mathrm{O}_{2}$

587 consumption rates with and without a microbial inoculum added (Supplementary Material

588 10). The inoculum was prepared from sediments collected with a shovel from a flowing reach

589 of the Albarine River close to Lyon, France ${ }^{14}$. We added $250 \mathrm{~mL}$ of Volvic ${ }^{\circledR}$ water to $250 \mathrm{~mL}$ 
of sediment and placed it twice in an ultrasonic bath (Branson 5510E, Emerson, MO, USA)

591 for $30 \mathrm{~s}$. The suspension of water and sediment was gently shaken after ultrasonication. We 592 then added $2.5 \mathrm{~mL}$ of the inoculum suspension to each respiration flask before filling them 593 with Volvic ${ }^{\circledR}$ water. Before adding the inoculum, the suspension was gently shaken again to

594 ensure a uniform inoculum distribution within the flask. In addition, we compared oxygen 595 consumption rates without inoculum by using stream water from three LL collection sites 596 (Albarine, Audeux and Calavon), instead of Volvic ${ }^{\circledR}$ mineral water (Supplementary

597 Material 10). We did not use an inoculum in our final experiments, because: a) it is 598 conceptually problematic to use an inoculum from one system to quantify the 599 decomposability of material from other areas and the large variability induced by doing so 600 could mask large-scale patterns of oxygen consumption rates upon rewetting; b) it was 601 impractical to ask international participants to send 2-3 L of river water to IRSTEA, 602 especially when the rivers were dry; c) it is virtually impossible to keep an inoculum constant 603 among runs in laboratory microcosms. By not adding an inoculum, our $\mathrm{O}_{2}$ consumption rates 604 were likely underestimated (i.e. conservative) relative to in-situ rates of $\mathrm{O}_{2}$ consumption 605 (Supplementary Material 10).

606

607 Data analysis. We used random forests (RFs) to explore relationships between environmental 608 variables and TPL quantity, LL decomposability, and $\mathrm{CO}_{2}$ release upon rewetting events. RFs 609 are highly flexible regression techniques suitable for modelling response variables (e.g., the 610 quantity and decomposability of TPL) that show complex relationships with environmental 611 variables (e.g., climate, riparian zone, flow regime, channel topography). RFs are invariant to 612 monotonic transformations of environmental variables, perform better than other regression 613 techniques when facing multicollinearity, are relatively robust to over-fitting, automatically fit 
614 non-linear relationships and high-order interactions, provide an overall goodness-of-fit

615 measure $\left(\mathrm{R}^{2}\right)$ and a measure of importance of each variable in a model ${ }^{48-50}$.

616

617 The role of environmental variables in RF models can be examined using importance

618 measures and partial dependence plots. Importance measures provide the contribution of

619 variables to model accuracy and are obtained from the degradation in model performance

620 when a predictor is randomly permuted ${ }^{48,50}$. Partial dependence plots show the marginal

621 contribution of a variable to the response (i.e., the response as a function of the variable when

622 the other variables are held at their mean value ${ }^{48-50}$ ) and were used to interpret the

623 relationships between predictors and dependent variables (responses), which were $\log _{10}(\mathrm{x}+1)$

624 transformed prior to analyses. Sets of global RF models were run for the main dependent

625 variables (quantities of TPL and LL; LL C:N, respiration rate and $\mathrm{CO}_{2}$ production) and then

626 these RF sets were run for each of three climate zones, using the Köppen-Geiger classification

627 of sampling sites: arid (merging Köppen-Geiger BSh, BSk, BWh and BWk; n=31), temperate

628 (merging Cfa, Cfb, Csa, Csb, Cwa; $\mathrm{n}=150$ ) and tropical (merging As, Aw; $\mathrm{n}=19$ ). No RF

629 models were run for alpine and continental climates due to the low number $(\leq 10)$ of sampling

630 sites.

631

632 We ran all global and climate-specific models with and without 'time since senescence' as a

633 predictor to assess the potential of this variable to improve predictive power, despite the large

634 uncertainty of this variable in some climate zones, particularly in the tropics. Removing the

635 variable from the models did not improve or diminish predictive power, including for IRES in

636 the tropics, but since RF models selected it as a strong predictor for most response variables,

637 we decided to include it in the analyses. The threshold to assess statistical significance was

6380.05 for all analyses, which were conducted in R 3.3.3 $3^{51}$ using the "RandomForest" package ${ }^{52}$. 
640 Data availability: The presented data are available on the FIGSHARE repository under the

643 Code availability: Not applicable.

\section{References}

646 38. Leopold, L. B. Channel and Hillslope Processes in a Semiarid Area, New Mexico. (Department of the Interior, U.S.A., 1966). Introduction for Ecologists. 2nd edn. (John Wiley \& Sons, 2004).

650 40. UNEP (United Nations Environment Programme). World Atlas of Desertification. 2nd edn. (UNEP, 1997).

651 41. Peel, M. C., Finlayson, B. L. \& McMahon, T. A. Updated world map of the Köppen-Geiger climate classification. Hydrol. Earth Syst. Sci. 11, 1633-1644 (2007).

42. Estiarte, M. \& Peñuelas, J. Alteration of the phenology of leaf senescence and fall in winter deciduous species by climate change: effects on nutrient proficiency. Glob. Change Biol. 21, 1005-1017 (2015).

43. Benton, T. G., Solan, M., Travis, J. M. \& Sait, S. M. Microcosm experiments can inform global ecological problems. Trends Ecol. Evol. 22, 516-521 (2007).

44. Mora-Gómez, J. et al. Microbial decomposition is highly sensitive to leaf litter emersion in a permanent temperate stream. Sci. Total Environ. 621, 486-496 (2018).

45. Dorca-Fornell, C. et al. Increased leaf mesophyll porosity following transient retinoblastoma-related protein silencing is revealed by microcomputed tomography imaging and leads to a system-level physiological response to the altered cell division pattern. Plant J. 76, 914-929 (2013).

662 46. Davidson, E. A. \& Janssens, I. A. Temperature sensitivity of soil carbon decomposition and feedbacks to climate change. Nature 440, 165-173 (2006).

664 47. Dilly, O. Microbial respiratory quotient during basal metabolism and after glucose amendment in soils and litter. Soil Biol. Biochem. 33, 117-127 (2001).

666 48. Pitcher, R. C. et al. Exploring the role of environmental variables in shaping patterns of seabed biodiversity 
668 49. Breiman, L. Random forests. Mach. Learn. 45, 5-32 (2001).

669 50. Leigh, C. \& Datry, T. Drying as a primary hydrological determinant of biodiversity in river systems: A 670 broad-scale analysis. Ecography 40, 487-499 (2017).

671 51. R Core Team. R: A Language and Environment for Statistical Computing (R Foundation for Statistical 672 Computing, 2017). Available at: http://www.R-project.org (last accessed: July 12017 ).

673 52. Liaw, A. \& Wiener, M. Classification and Regression by Random Forest. R News 2, 18-22 (2002). 674 